\title{
Defizite und Perspektiven
}

\author{
Experten befassten sich mit der aktuellen Situation.
}

Trotz zahlreicher Appelle sind in der Kinder- und Jugendpsychiatrie sowohl ambulant, als auch stationär nach wie vor eklatante Defizite zu beklagen. Immer mehr Kinder und Jugendliche benötigen jedoch psychiatrische Betreuung. Laut Österreichischem Strukturplan Gesundheit (ÖSG) bräuchte Wien 128 bis 208 Betten für Kinderund Jugendpsychiatrie. Die aktuellen Zahlen sehen jedoch anders aus: Derzeit gibt es in Wien nur 56 Betten und 20 Tagesklinik-Plätze.

Im März befasste sich ein Pressegespräch auf Einladung der Volkanwaltschaft mit diesen Problemen. Die Teilnehmer: Dr. Günther Kräuter, Vorsitzender derVolksanwaltschaft, Univ.-Prof. Dr. Ernst Berger, Kinder- und Jugendpsychiater und Leiter der Kommission 4 der Volksanwaltschaft, Dr. Sigrid Pilz, Leiterin der Wiener Pflege-, Patientinnen- und Patientenanwaltschaft und Mag. Bernd Rappert, Bereichsleiter von VertretungsNetz/Patientenanwaltschaft.

\section{Kinder sind keine kleinen Erwachsenen!}

Die geringe Versorgungsdichte führt dazu, dass durchschnittlich ein bis zwei Kinder und Jugendliche im Alter zwischen 12 und 17 Jahren in Wien auf der stationären Erwachsenenpsychiatrie untergebracht sind. Die Konfrontation mit psychisch erkrankten Erwachsenen ist für Minderjährige allerdings massiv belastend. Auf der Erwachsenenpsychiatrie fehlen zudem eine altersadäquate Betreuung, ein pädagogisches Angebot und das Zusammensein in einer Gruppe Gleichaltriger. Auch das Landesgericht Wien hat klargestellt, dass Minderjährige ein Recht darauf haben, in einer Spezialstation für Kinder- und Jugendpsychiatrie untergebracht zu werden.

\section{Statements}

Dr. Günther Kräuter - Fehlende Transitionspsychiatrie

"Die Unterbringung von Kindern und Jugendlichen in der Erwachsenenpsychiatrie ist ein eklatanter Missstand und muss möglichst rasch beendet werden. Zusätzlich müssen dringend Angebote für junge, psychisch kranke Menschen zwischen 16 und 25 Jahren entwickelt werden, die den Besonderheiten des Übergangs von der Jugend ins Erwachsenenalter gerecht werden."

\section{Univ. Prof. Dr. Ernst Berger - Tageskliniken und sozialtherapeutische Wohnplätze fehlen}

„Ein Ausbau tagesklinischer Behandlungsplätze ist bedeutend kostengünstiger als die Einrichtung einer Krankenhausstation. Überdies ist tagesklinische Behandlung dem Lebensalltag der Patienten bedeutend näher. Nach wie vor besteht ein deutliches Defizit an sozialtherapeutischen Wohnplätzen."

\section{Dr. Sigrid Pilz - Aufstockung von Betten und Fachärzten gefordert}

„2016 mussten in Wien 163 Kinder und Jugendliche auf der stationären Erwachsenenpsychiatrie aufgenommen werden, weil nicht genügend Betten in der Kinderund Jugendpsychiatrie zur Verfügung stehen."

\section{Mag. Bernhard Rappert - Altersadäquate Betreuung}

„Der Aufenthalt auf der Erwachsenenpsychiatrie ist für Jugendliche extrem belastend. Sie erhalten dort keine ihrem Alter entsprechende Betreuung, es kommt durch altersinadäquate Abläufe zu Traumatisierungen, in suizidalen Krisen fehlen Vertrauenspersonen."

\section{Forderungen der Experten}

- Behandlung von Minderjährigen ausschließlich auf Stationen für Kinder- und Jugendpsychiatrie.

- Die Bettenkapazitäten in der Kinder- und Jugendpsychiatrie sind rasch zu erweitern.

- Ambulante und tagesklinische Strukturen sind zu verstärken.

- Geeignete Angebote für junge psychisch erkrankte Menschen zwischen 16 und 25 Jahren sind zu entwickeln.

- Zusätzliche Kassenvertragsfacharztstellen sind dringend erforderlich.

- Erhöhung und zeitnahe Besetzung offener Ausbildungsplätze im Fach Kinder- und Jugendpsychiatrie hat zu erfolgen.
Paediatr. Paedolog. Austria 2017 · 52:113 DOI 10.1007/s00608-017-0480-2

๑) Springer-Verlag Wien 2017

Quelle: Pressegespräch der Volksanwaltschaft, 23.03.2017

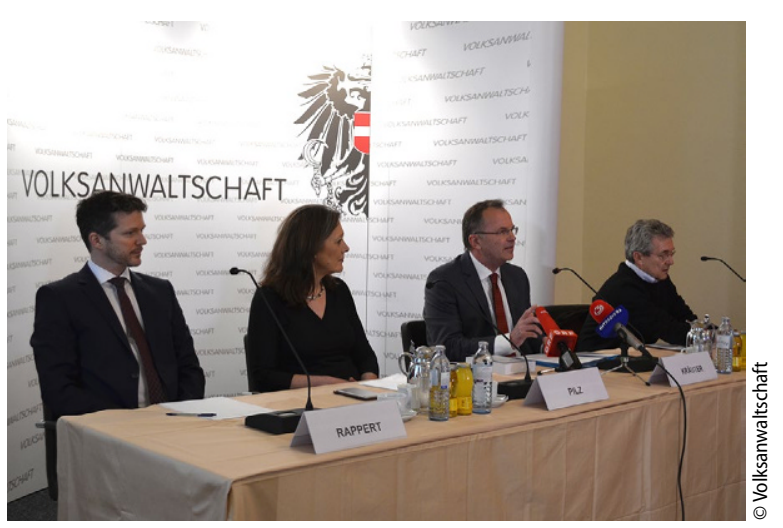

$\Delta$ V.I.n. r.: B. Rappert, S. Pilz, G. Kräuter, E. Berger 